\title{
Baryogenesis in false vacuum
}

\author{
Yuta Hamada ${ }^{1, a}$, Masatoshi Yamada ${ }^{2, b}$ \\ ${ }^{1}$ KEK Theory Center, IPNS, KEK, Tsukuba, Ibaraki 305-0801, Japan \\ 2 Institute for Theoretical Physics, Kanazawa University, Kanazawa 920-1192, Japan
}

Received: 14 June 2017 / Accepted: 11 September 2017 / Published online: 23 September 2017

(C) The Author(s) 2017. This article is an open access publication

\begin{abstract}
The null result in the LHC may indicate that the standard model is not drastically modified up to very high scales, such as the GUT/string scale. Having this in the mind, we suggest a novel leptogenesis scenario realized in the false vacuum of the Higgs field. If the Higgs field develops a large vacuum expectation value in the early universe, a lepton number violating process is enhanced, which we use for baryogenesis. To demonstrate the scenario, several models are discussed. For example, we show that the observed baryon asymmetry is successfully generated in the standard model with higher-dimensional operators.
\end{abstract}

\section{Introduction}

Although the standard model (SM) is complete after the discovery of the Higgs boson at the Large Hadron Collider [1,2], there are still mysteries in elementary particle physics, such as the finite neutrino mass and dark matter. Besides these the baryon asymmetry in the universe (BAU) is also one of the unsolved problems. That is, how has baryogenesis been realized in the evolution of the universe? The latest cosmological result from the Planck observations [3] tells us that the BAU is

$\frac{n_{B}}{s}=(8.67 \pm 0.05) \times 10^{-11}$,

where $n_{B}$ is the baryon number density and $s$ is the entropy density.

In order to theoretically explain the BAU within elementary particle physics, the Sakharov conditions [4] have to be satisfied: there exists a process violating the baryon number conservation; $C$ and $C P$ invariances are violated; the system leaves its equilibrium state. The SM does not accommodate the departure from equilibrium. Although the baryon number

\footnotetext{
a e-mail: yhamada@post.kek.jp

b e-mail: masay@hep.s.kanazawa-u.ac.jp
}

is violated through the sphaleron process and $C P$ symmetry is violated in the weak interaction, it is not enough to reproduce the BAU. Therefore the SM cannot satisfy these conditions and must be extended.

Some baryogenesis mechanisms satisfying the Sakharov conditions have been suggested, e.g. the grand unified theory [5] and the Affleck-Dine mechanism [6]. Leptogenesis is also one of the well-known mechanisms for baryogenesis [7] (see also the reviews $[8,9]$ ) where we use the fact that through the sphaleron process [10-13], the difference $B-L$ between the baryon number $B$ and the lepton number $L$ is conserved whereas their sum $B+L$ is not. The baryon number density in thermal equilibrium is provided by the $B-L$ number density via the sphaleron process:

$n_{B}=\frac{8 N_{F}+4 N_{S}}{22 N_{F}+13 N_{S}} n_{B-L}$,

where $N_{F}$ is generation of quarks and leptons and $N_{S}$ is that of scalar doublets. For instance, in the case of the SM where $N_{F}=3$ and $N_{S}=1$, the factor in the right-hand side is 28/79. Through the decay of the heavy particle, the lepton number is generated, and then its number density changes to the $B-L$ number density $n_{B-L}$, whose process is described by the coupled Boltzmann equations for these number densities.

In this paper, we study leptogenesis realized in the false vacuum of the Higgs field, in which the Higgs gains a vacuum expectation value far above the electroweak scale. The mass of the particles coupled to the Higgs field becomes supermassive, and the left-handed neutrinos can become heavier than the charged leptons and $W$ boson in the presence of higher-dimensional lepton number violating operators. The decay of the left-handed neutrinos creates an $L$ asymmetry. Shortly after, the phase transition of the Higgs takes place and the Higgs moves from the false vacuum to the true electroweak one (where thermal effects restore the electroweak symmetry allowing the sphalerons to reprocess the $L$ into a $B$ asymmetry). We consider the situation where the right- 
handed neutrino masses are large compared with the reheating temperature. Therefore our scenario gives an alternative scenario for baryogenesis.

To demonstrate this scenario, two models are investigated. We first consider a minimal model depending on the SM with a high-dimensional operator,

$\Delta \mathcal{L}_{5}=\frac{\lambda_{i j}}{\Lambda} H H \bar{L}_{j}^{c} L_{i}$

where $L_{i}$ is the lepton doublet, $\Lambda$ is a cutoff scale, ${ }^{1}$ and the Higgs doublet is defined as

$H=\frac{1}{\sqrt{2}}\left(\begin{array}{c}\chi_{1}+i \chi_{2} \\ h+i \chi_{3}\end{array}\right)$

Such an operator is typically generated in the type I seesaw model by integrating out the right-hand neutrino. This effective interaction breaks the lepton number conservation and thus is used as the source of the lepton asymmetry. In particular, we consider the decays of the left-hand neutrino, given by the modes $v \rightarrow \ell^{-} W^{+}, \ell^{+} H^{-}, \ell^{-} H^{+}$. Note that in the broken phase $\langle H\rangle \neq 0$, the operator (3) turns into a neutrino mass term,

$\frac{\langle H\rangle^{2}}{\Lambda}\left(\bar{v}^{c} v+\bar{v} v^{c}\right)=\frac{\langle h\rangle^{2}}{2 \Lambda}\left(\bar{v}^{c} v+\bar{v} v^{c}\right)$,

where we have assumed that the coupling constant $\lambda_{i j}$ is of order one since neutrino can have a finite mass $m_{v} \sim 0.1 \mathrm{eV}$. Then the cutoff scale $\Lambda$ is estimated as

$\Lambda \simeq 6.0 \times 10^{14} \mathrm{GeV}\left(\frac{0.1 \mathrm{eV}}{m_{v}}\right)$.

Here, leptogenesis takes place in the false vacuum where the neutrino mass $\langle h\rangle^{2} / \Lambda$ becomes larger than the charged lepton and the $W$ boson ones. As will be seen in the next section, in such a minimal model, the baryon asymmetry produced by this process actually is not adequate for the observed value (1).

Next, we consider an extended system in which the new higher-dimensional operators are added. In this case, we will see that the lepton asymmetry is caused by the neutrino and it is possible to explain the observation.

We have to see whether or not the phase transition of the Higgs field from the false vacuum to the electroweak one occurs after the lepton asymmetry is produced. To this end, we investigate the thermal history of the Higgs potential. Including a new singlet-scalar field coupled to the SM Higgs

\footnotetext{
${ }^{1}$ In Refs. [14,15], the operator (3) is used to realize leptogenesis as well as the $C P$ violating operator $\bar{L}_{i} \gamma^{\mu} L_{j} \bar{L}_{i} \gamma_{\mu} L_{j}$. These operators are naturally generated in the low energy effective theories of various seesaw models.
}

field, there exists a certain parameter space where the phase transition appropriately takes place.

We organize this paper as follows: in the next section, we present the formulation of the Boltzmann equations in order to calculate the baryon asymmetry. Numerically solving them, we investigate the produced baryon asymmetry for two cases explained above. Section 3 is devoted to an investigation of the thermal history of the Higgs potential. We summarize and discuss our study and the results obtained, and we comment on the possibility of the high scale electroweak baryogenesis in Sect. 4. In Appendix A, the thermal effects on the Higgs potential and their formulations are shown.

\section{Mechanism and Boltzmann equations}

First, we consider a situation where the decay of the lefthanded neutrino produces the baryon asymmetry. In this section, we present the Boltzmann equations and quantitatively evaluate the baryon asymmetry by numerically solving them. We evaluate the baryon asymmetry produced by the left-handed neutrino decay; however, we see that not enough baryon asymmetry is produced. To ameliorate the situation, next we add the new higher-dimensional operators. We demonstrate that, in this case, the decay of the neutrino can reproduce the observed amount of asymmetry.

\subsection{The derivation of Boltzmann equations}

In this subsection, to calculate the asymmetry of the universe, we follow Refs. $[9,16,17]$ and derive the Boltzmann equations for the general case of leptogenesis. The change of the number density of a heavy particle is governed by

$$
\begin{aligned}
\dot{n}_{X}+3 H n_{X}= & \int \mathrm{d} \Pi_{X} \mathrm{~d} \Pi_{1} \mathrm{~d} \Pi_{2}(2 \pi)^{4} \delta^{(4)}\left(p_{X}-p_{1}-p_{2}\right) \\
& \times\left(-f\left(p_{X}\right)|\mathcal{M}(X \rightarrow 12)|^{2}\right. \\
& \left.+f\left(p_{1}\right) f\left(p_{2}\right)|\mathcal{M}(12 \rightarrow X)|^{2}\right) \\
& +\int \mathrm{d} \Pi_{X} \mathrm{~d} \Pi_{Y} \mathrm{~d} \Pi_{1} \mathrm{~d} \Pi_{2} \cdots \mathrm{d} \Pi_{N}(2 \pi)^{4} \delta^{(4)} \\
& \times\left(p_{X}+p_{Y}-p_{1}-p_{2}-\cdots-p_{N}\right) \\
& \times\left(-f\left(p_{X}\right) f\left(p_{Y}\right)|\mathcal{M}(X Y \rightarrow 12 \cdots N)|^{2}\right. \\
& \left.+f\left(p_{1}\right) \cdots f\left(p_{N}\right)|\mathcal{M}(12 \cdots N \rightarrow X Y)|^{2}\right),
\end{aligned}
$$

where $X$ and $Y$ represent the heavy particles; the numbers $1 \cdots N$ denote lighter particles; the dot on $n_{X}$ in the lefthand side denotes the time derivative; we have neglected the effects of the Pauli blocking and stimulated emission; $\mathrm{d} \Pi_{i}=\mathrm{d}^{3} p_{i} /(2 \pi)^{3} 2 E_{i}$ is the phase space integral; $H=$ $\dot{R} / R$ is the Hubble parameter given by the scale factor $R$, 
which is governed by the Friedmann equation. Here $f$ is the distribution function, approximately given by the MaxwellBoltzmann distribution.

The first and second terms of the right-hand side in Eq. (7) correspond to the decay and annihilation of heavy particles, respectively. Let us rewrite the first term by using the definition of the decay rate,

$\Gamma_{X} \equiv \frac{1}{2 E_{X}} \int \mathrm{d} \Pi_{1} \mathrm{~d} \Pi_{2}(2 \pi)^{4} \delta^{(4)}\left(p_{X}-p_{1}-p_{2}\right)|\mathcal{M}(X \rightarrow 12)|^{2}$.

We use the fact that the kinetic equilibrium allows us to make the replacement, ${ }^{2}$

$$
f\left(p_{1}\right) f\left(p_{2}\right)=f^{\mathrm{EQ}}\left(p_{1}\right) f^{\mathrm{EQ}}\left(p_{2}\right)=f^{\mathrm{EQ}}\left(p_{X}\right) .
$$

Furthermore, at leading order, $|\mathcal{M}(X \rightarrow 12)|^{2}=\mid \mathcal{M}(12 \rightarrow$ $X)\left.\right|^{2}$. Hence, we find that the first term in the right-hand side becomes

$\left(-n_{X}+n_{X}^{\mathrm{EQ}}\right) \Gamma_{X}$

The second term in Eq. (7) can be written in terms of the thermal average cross section of the pair annihilation $\left\langle\sigma_{\mathrm{ann}} v\right\rangle$ :

where the first and second terms in the right-hand side describe the decay of the heavy particle and annihilation of the leptons, respectively; $W$ is a particle without the lepton number; $l_{i}$ is a particle having the lepton number; the process $X \leftrightarrow l W$ breaks the lepton number symmetry. Furthermore, we rewrite this equation as one for $B-L$ asymmetry, which is given by

$$
\begin{aligned}
\dot{n}_{B-L}+3 H n_{B-L}= & -\epsilon\left(n_{X}-n_{X}^{\mathrm{EQ}}\right) \Gamma_{X} \mathrm{Br}-n_{B-L} \Gamma_{X} \mathrm{Br} \frac{n_{X}^{\mathrm{EQ}}}{n_{\gamma}} \\
& -2 n_{B-L} n_{l}\left\langle\sigma_{L} v\right\rangle
\end{aligned}
$$

where $\epsilon$ is the parameter which denotes the $C P$ asymmetry; $\mathrm{Br}$ is the branching ratio of $X \rightarrow l W ; n_{\gamma}$ is the number density of photon; and $\left\langle\sigma_{L} v\right\rangle$ is the thermally-averaged scattering cross section which does not conserve the lepton number.

It is convenient to introduce $N_{i} \equiv n_{i} / n_{\gamma}$ because this quantity is conserved under the cosmic expansion. We also introduce $z \equiv M_{X} / T$ as a variable. Using these variables, let us now rewrite the Boltzmann equations. For instance, the left-hand side becomes

$\dot{n}_{X}+3 H n_{X}=n_{\gamma} \dot{N}_{X}=n_{\gamma} H z \frac{\mathrm{d}}{\mathrm{d} z} N_{X}$,

We assume that $f\left(p_{i}\right) \propto f^{\mathrm{EQ}}\left(p_{i}\right)$ thanks to the kinetic equilibrium, so that the second term in Eq. (7) becomes

$\left\langle\sigma_{\mathrm{ann}} v\right\rangle\left(-n_{X}^{2}+\left(n_{X}^{\mathrm{EQ}}\right)^{2}\right)$

To summarize, the Boltzmann equation of $n_{X}$ is given by

$$
\dot{n}_{X}+3 H n_{X}=\left(-n_{X}+n_{X}^{\mathrm{EQ}}\right) \Gamma_{X}+\left\langle\sigma_{\mathrm{ann}} v\right\rangle\left(-n_{X}^{2}+\left(n_{X}^{\mathrm{EQ}}\right)^{2}\right) .
$$

In a similar manner, we can write the Boltzmann equation governing the lepton number density:

$$
\begin{aligned}
\dot{n}_{l} & +3 H n_{l}=\int \mathrm{d} \Pi_{X} \mathrm{~d} \Pi_{l} \mathrm{~d} \Pi_{W} \delta^{(4)}\left(p_{X}-p_{l}-p_{W}\right) \\
& \times \epsilon\left(-f\left(p_{X}\right)|\mathcal{M}(X \rightarrow l W)|^{2}\right. \\
& \left.+f\left(p_{l}\right) f\left(p_{W}\right)|\mathcal{M}(l W \rightarrow X)|^{2}\right) \\
& +2 \int \mathrm{d} \Pi_{1} \mathrm{~d} \Pi_{2} \mathrm{~d} \Pi_{3} \mathrm{~d} \Pi_{4} \delta^{(4)}\left(p_{1}+p_{2}-p_{3}-p_{4}\right) \\
& \times\left(-f\left(p_{1}\right) f\left(p_{2}\right)\left|\mathcal{M}\left(l_{1} l_{2} \rightarrow l_{3} l_{4}\right)\right|^{2}\right. \\
& \left.+f\left(p_{3}\right) f\left(p_{4}\right)\left|\mathcal{M}\left(l_{3} l_{4} \rightarrow l_{1} l_{2}\right)\right|^{2}\right),
\end{aligned}
$$

$\overline{2}$ Here we neglect the chemical potential of particle 1 and 2 as the effect is subleading. where, in the second equality, we have used

$$
\frac{\mathrm{d} T}{\mathrm{~d} t}=-3 H \frac{n_{\gamma}}{\mathrm{d} n_{\gamma} / \mathrm{d} T}=-H T .
$$

The right-hand side is

$$
\begin{aligned}
- & \Gamma_{X}(z)\left(n_{X}-n_{X}^{\mathrm{EQ}}\right)-\left\langle\sigma_{\mathrm{ann}} v\right\rangle\left(n_{X}^{2}-\left(n_{X}^{\mathrm{EQ}}\right)^{2}\right) \\
= & -n_{\gamma} H z\left(\frac{\Gamma_{X}(z)}{H(z) z}\right)\left(N_{X}-N_{X}^{\mathrm{EQ}}\right) \\
& -n_{\gamma} H z\left(\frac{\left\langle\sigma_{\mathrm{ann}} v\right\rangle n_{\gamma}}{H(z) z}\right)\left(N_{X}^{2}-\left(N_{X}^{\mathrm{EQ}}\right)^{2}\right) .
\end{aligned}
$$

In terms of $N_{i}$ and $z$, we can write the set of the Boltzmann equations as follows:

$$
\begin{aligned}
\frac{\mathrm{d}}{\mathrm{d} z} N_{X}= & -\left(\frac{\Gamma_{X}(z)}{H(z) z}\right)\left(N_{X}-N_{X}^{\mathrm{EQ}}\right) \\
& -\left(\frac{\left\langle\sigma_{\mathrm{ann}} v\right\rangle n_{\gamma}}{H(z) z}\right)\left(N_{X}^{2}-\left(N_{X}^{\mathrm{EQ}}\right)^{2}\right), \\
\frac{\mathrm{d}}{\mathrm{d} z} N_{B-L}= & -\left(\frac{\epsilon \Gamma_{X}(z) \mathrm{Br}}{H(z) z}\right)\left(N_{X}-N_{X}^{\mathrm{EQ}}\right) \\
& -N_{B-L}\left(\frac{\Gamma_{X}(z) \mathrm{Br}}{H(z) z}\right) N_{X}^{\mathrm{EQ}}
\end{aligned}
$$


$-\left(\frac{\left\langle\sigma_{L} v\right\rangle n_{\gamma}}{H(z) z}\right) 2 N_{B-L} N_{l}$

$H^{2}(z)=\frac{\pi^{2} g_{*}(z)}{90} \frac{z^{4} M_{X}^{4}}{M_{P}^{2}}$,

$N_{X}^{\mathrm{EQ}}=\frac{g}{4 \zeta(3)} z^{2} K_{2}(z)$

$n_{\gamma}=\frac{2 \zeta(3)}{\pi^{2}} T^{3}=\frac{2 \zeta(3)}{\pi^{2}} z^{-3} M_{X}$

where $\zeta(3) \approx 1.20205$ is the Riemann zeta function of 3 ; $M_{P}=\sqrt{\hbar c / 8 \pi G}=2.435 \times 10^{18} \mathrm{GeV}$ is the reduced Planck scale; $K_{2}$ is for the modified Bessel functions of the second kind; $g_{*}(z)$ is the total number of effectively massless degrees of freedom; and $g$ is the internal degrees of freedom of the heavy particle. We neglect the $z$ dependence of $g_{*}(z)$ and use $g_{*}=106.75$.

Simultaneously solving the Boltzmann equations, we can evaluate the value of the lepton asymmetry due to the decay of the left-handed neutrino which is identified with the heavy particle $X$. In order to perform numerical calculations, we have to specify $\Gamma_{X}, \mathrm{Br}, \epsilon,\left\langle\sigma_{\mathrm{ann}} v\right\rangle$ and $\left\langle\sigma_{L} v\right\rangle$. In the next subsection, we give these variables for the minimal model.

\subsection{Minimal model case}

We evaluate the baryon asymmetry in the minimal model whose Lagrangian is given as

$\mathcal{L}=\mathcal{L}_{\mathrm{SM}}+\Delta \mathcal{L}_{5}$

where $\mathcal{L}_{\mathrm{SM}}$ is the Lagrangian of the SM and $\Delta \mathcal{L}_{5}$ is the higher-dimensional operator given in Eq. (3). The lepton number is produced by the decay of the left-handed neutrino. We now show the variables given in the Boltzmann equations in order.

The masses of the left-handed neutrino, the Higgs boson and the $W$ boson in the broken phase $\langle H\rangle \neq 0$ are given as

$m_{\nu}=\frac{\langle h\rangle^{2}}{\Lambda}, \quad M_{H}=\sqrt{2 \lambda}\langle h\rangle, \quad M_{W}=\frac{1}{2} g_{2}\langle h\rangle$,

respectively, where $\lambda$ is the quartic coupling constant of the Higgs field, and $g_{2}$ is the $\mathrm{SU}(2)_{L}$ gauge coupling constant.

The decay rate of the left-handed neutrino, $v_{i}$, and the branching ratio to the longitudinal gauge boson are calculated $\mathrm{as}^{3}$

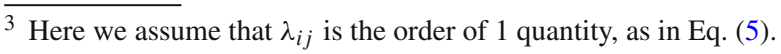

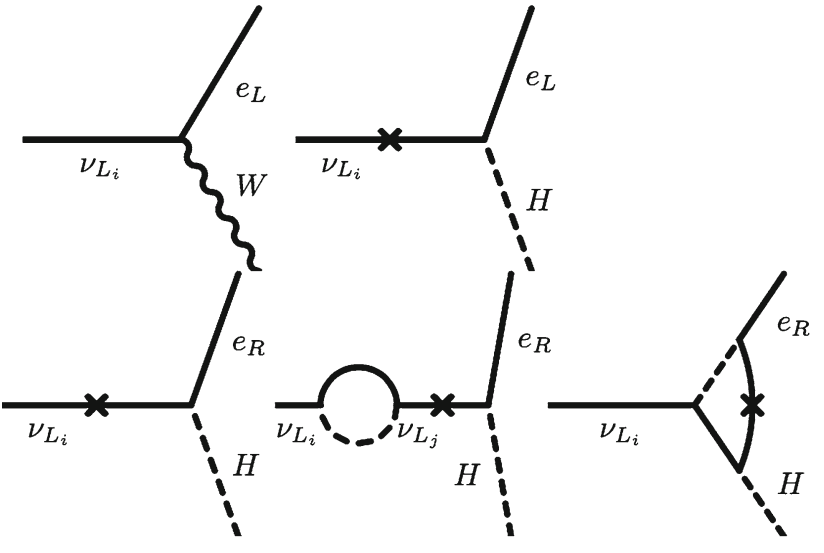

Fig. 1 The first two diagrams are the main decay modes of the neutrino where the second one comes from the vertex $\langle H\rangle H \bar{L}_{j}^{c} L_{i}$. The last three diagrams contribute to the asymmetry by the decay of the left-hand neutrino. The complex phase appears only if the mass of the neutrino is larger than that of the $W$ boson. The Majorana mass term for the left-handed neutrino explicitly breaks the lepton number conservation, which is represented by a cross symbol in the diagram

$$
\begin{aligned}
\Gamma_{X}(z)= & \left.\left\langle\frac{1}{\gamma}\right\rangle \Gamma_{X}\right|_{z=\infty} \simeq\left\langle\frac{1}{\gamma}\right\rangle \frac{m_{v}}{8 \pi} \\
& \times\left(\left(\lambda_{i i} \frac{\langle h\rangle}{\sqrt{2} \Lambda}\right)^{2}+g_{2}^{2}+y_{\tau}^{2}\right), \\
\operatorname{Br} \simeq & \frac{y_{\tau}^{2}}{\left(\frac{\langle h\rangle}{\sqrt{2} \Lambda}\right)^{2}+g_{2}^{2}},
\end{aligned}
$$

where $\langle 1 / \gamma\rangle=K_{1}(z) / K_{2}(z)$ in the thermal bath, $K_{1}$ is for the modified Bessel functions of the first kind, $\lambda_{i i}$ is diagonalized by rotating the lepton field, and $y_{\tau}$ is the tau Yukawa coupling. We note that the branching ratio to the transverse gauge boson is important. This is because, in order to pick up the imaginary part of the amplitude, one needs to use the lepton Yukawa coupling rather than the SU(2) gauge coupling. 4

The $C P$ asymmetry $\epsilon$ comes from the interference between the tree and the loop diagrams corresponding to the last three diagrams in Fig. 1, whose order is given by

$\epsilon_{i} \simeq \frac{1}{8 \pi} \sum_{j} \frac{\operatorname{Im}\left[\left(Y Y^{\dagger}\right)_{i j}^{2}\right]}{y_{\tau}^{2}}$

where $Y$ is the charged lepton Yukawa matrix. Note that the imaginary part appears only if $m_{v}>M_{W}+M_{\tau}{ }^{5}$ which yields

\footnotetext{
$\overline{4}$ The coupling $\lambda_{i j}$ is not helpful in obtaining the imaginary part because this coupling becomes real by rotating the lepton field.

${ }^{5}$ Even if $m_{v}<M_{W}+M_{\tau}$, the imaginary part appears in higher order. However, it is too small to obtain sufficient baryon asymmetry.
} 
$\langle h\rangle>\frac{g_{2}}{2} \Lambda \simeq 1.5 \times 10^{14} \mathrm{GeV}\left(\frac{\Lambda}{6 \times 10^{14} \mathrm{GeV}}\right)$.

Here $M_{\tau}$ is the mass of the tau lepton.

Let us estimate the imaginary parts of the Yukawa coupling constants in Eq. (27). The numerator of Eq. (27) is related to the Jarlskog invariant in the lepton sector [18], and the order is estimated as [18]

$\epsilon_{i} \sim \frac{1}{8 \pi} y_{\tau}^{2}\left(3 \times 10^{-2} \sin \delta\right) \simeq 1.2 \times 10^{-7}\left(\frac{y_{\tau}}{10^{-2}}\right)^{2}\left(\frac{\sin \delta}{1}\right)$,

where $\delta$ is the Dirac CP phase of the neutrino sector.

We note that, by using the renormalization group equations, we obtain the values of the coupling constants at the high scale: ${ }^{6}$

$g_{2} \simeq 0.5, \quad y_{\tau} \simeq 1 \times 10^{-2}, \quad \alpha_{2} \simeq \frac{g_{2}^{2}}{4 \pi}=\frac{1}{50}$.

\section{Numerical result in minimal model}

The Planck observation [3] tells us

$N_{B, \mathrm{obs}} \simeq 6.1 \times 10^{-10} \times \frac{2387}{86}=1.7 \times 10^{-8}$,

where the factor $2387 / 86$ is the photon production factor. ${ }^{7}$ If this value comes from the sphaleron effect, we shouldhave

$N_{B-L, \text { obs }} \simeq 6.1 \times 10^{-10} \times \frac{2387}{86} \times \frac{79}{28}=4.8 \times 10^{-8}$.

Therefore, we numerically solve the Boltzmann equations given in Eqs. (19)-(23) and investigate whether or not the appropriate parameter space which satisfies the value (33) exists.

Unfortunately, we can easily see that the baryon asymmetry cannot be reproduced in this framework. We obtain

\footnotetext{
${ }^{6}$ See e.g. Ref. [19].

7 This factor comes from the ratio $g_{* S}\left(T_{B}\right) / g_{* S}$ (today), where $g_{* S}$ is the effective degrees of freedom for the entropy, and $T_{B}$ is the temperature of the baryogenesis. Here $g_{* s}$ is given by
}

$g_{* s}($ today $)=\frac{43}{11}, \quad g_{* s}\left(T_{B}\right)=\frac{217}{2}$.

The photon and the left-handed neutrinos contribute $g_{* S}$ (today), and we assume that the SM particles and one generation right-handed neutrino contributes $g_{* s}\left(T_{B}\right)$. Even if the right-handed neutrino contribution is absent, this factor rarely changes.

$$
\begin{aligned}
\epsilon_{i} \mathrm{Br} & \sim 1.2 \times 10^{-7} \frac{y_{\tau}^{2}}{\left(\frac{\langle h\rangle}{\sqrt{2} \Lambda}\right)^{2}+g_{2}^{2}}\left(\frac{y_{\tau}}{10^{-2}}\right)^{2}\left(\frac{\sin \delta}{1}\right) \\
& \lesssim 2 \times 10^{-10}
\end{aligned}
$$

by combining Eqs. (26) and (29), and hence the resultant baryon asymmetry is too small to explain the current data. This indicates the necessity of an extension of the model. In the next subsection, we present the possible extension to realize the observed baryon asymmetry.

\subsection{Extended model}

A way to improve the situation is to add new operator. The smallness of the charged lepton Yukawa coupling results in the small baryon asymmetry. Therefore, if this coupling is modified in the false vacuum, the situation changes. Let us assume the existence of the higher-dimensional operator which contributes as the Yukawa coupling in the vacuum where Higgs takes the large VEV:

$y_{2, i j} \frac{H^{\dagger} H}{\Lambda_{2}^{2}} \bar{E}_{i} H L_{j}+$ h.c.

Similar, we consider the operator which gives the correction to Majorana neutrino masses:

$y_{3, i j} \frac{H^{\dagger} H}{\Lambda_{3}^{3}} H H \bar{L}_{j}^{c} L_{i}+$ h.c.

In general, $\Lambda_{3}$ can be different from $\Lambda_{1}$ in Eq. (3). This structure may occur when we consider the right-handed neutrino model as a UV completion for example, ${ }^{8}$ where the action is

$M_{N, i j} \bar{N}_{i}^{c} N_{j}+\left(y_{N i j}+y_{N 2, i j} \frac{H^{\dagger} H}{\Lambda_{N 2}^{2}}\right) \bar{N}_{i} H L_{j}+$ h.c.

We evaluate the order of the resultant asymmetry obtained by the decay of the SM neutrino again. The set of the thermal initial conditions of the Boltzmann equations is

$N_{X}\left(z_{\text {ini }}\right)=\frac{3}{4}, \quad N_{B-L}\left(z_{\text {ini }}\right)=0$.

In this case, the decay rate is ${ }^{9}$

\footnotetext{
8 This is just a possibility of the UV completion, and we do not insist on this model in the following discussion.

${ }^{9}$ The last factor appears because of the phase space integral.
} 

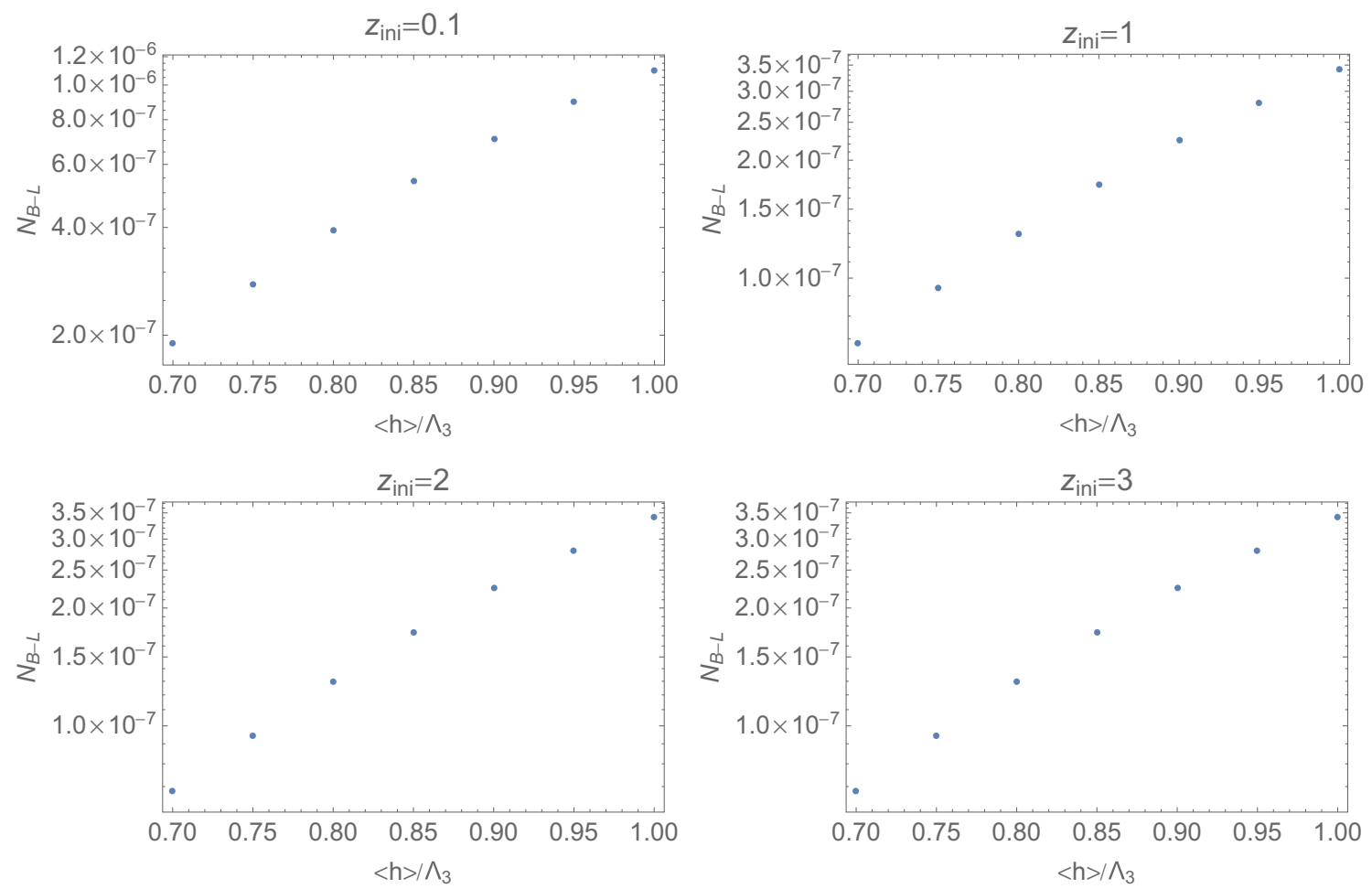

Fig. $2 N_{B-L}$ created by the decay of the neutrinos in the presence of the higher-dimensional operators. The parameters are taken as in Eq. (41)

$$
\begin{aligned}
\Gamma_{X}(z)= & \left\langle\frac{1}{\gamma}\right\rangle \frac{M_{v}}{8 \pi}\left(g_{2}^{2}+\left|Y_{2}\right|^{2}+\frac{\langle h\rangle^{2}}{2 \Lambda^{2}}+\left(\frac{\langle h\rangle^{3}}{2 \sqrt{2} \Lambda_{3}^{3}}\right)^{2}\right) \\
& \times \sqrt{1-\left(\frac{M_{W}^{2}}{M_{v}^{2}}\right)},
\end{aligned}
$$

and the functions which appear in Boltzmann equation are roughly given by

$$
\begin{aligned}
& \epsilon_{i} \simeq \sum_{j} \frac{\operatorname{Im}\left[\left(Y_{2} Y_{2}^{\dagger}\right)^{2}\right]_{i j}}{8 \pi\left(Y_{2} Y_{2}^{\dagger}\right)_{i j}}, \\
& \left\langle\sigma_{\mathrm{ann}} v\right\rangle \simeq \alpha_{2}^{2} \frac{1}{\operatorname{Max}\left(M_{v}^{2}, T^{2}\right)}, \\
& \left\langle\sigma_{L} v\right\rangle \simeq\left(\frac{\left|Y_{2}\right|^{2}}{4 \pi}\right)^{2} \frac{1}{\operatorname{Max}\left(M_{v}^{2}, T^{2}\right)}, \\
& \operatorname{Br} \simeq \frac{1}{\frac{g_{2}^{2}}{\left|Y_{2}\right|^{2}}+1+\frac{\langle h\rangle^{2}}{2\left|Y_{2}\right|^{2} \Lambda^{2}}+\left(\frac{\langle h\rangle^{3}}{2 \sqrt{2}\left|Y_{2}\right| \Lambda_{3}^{3}}\right)^{2}},
\end{aligned}
$$

where we define the effective charged lepton Yukawa coupling $Y_{2 i j}:=Y_{i j}+y_{2, i j}\langle h\rangle^{2} /\left(2 \Lambda_{2}^{2}\right)$, and the neutrino mass $M_{\nu i}:=\operatorname{diag}\left(\lambda_{i j}\langle h\rangle^{2} / \Lambda+y_{3, i j}\langle h\rangle^{4} /\left(2 \Lambda_{3}^{3}\right)\right)_{i}$. We further assume that the components of $M_{v i}$ and $Y_{2 i j}$ are the same order of magnitude, respectively, and we denote $M_{v i}=M_{\nu}, Y_{2 i j}=Y_{2}$ for simplicity. We focus on the asym- metry generated by the lightest neutrino in the false vacuum. In Fig. 2, we show the result assuming that $C P$ phase is of the order of one, i.e. $\mathrm{e}^{i \delta} \sim 1$. We use the following parameter set to draw the plot:

$$
\begin{aligned}
\Lambda & =6 \times 10^{14} \mathrm{GeV}, \quad \Lambda_{2}=6 \times 10^{13} \mathrm{GeV}, \\
\Lambda_{3} & =3 \times 10^{13} \mathrm{GeV}, \quad\langle h\rangle=2 \times 10^{13} \mathrm{GeV}, \\
y_{2 i j} & \simeq 1, \quad y_{3, i j}=1 .
\end{aligned}
$$

We can see that the BAU is reproduced in this extension.

Notice that, unlike the minimal model, we obtain

$\epsilon_{i} \mathrm{Br} \sim 3 \times 10^{-6}$.

In the extended model with the parameters in Eq. (41). This value is much larger than that in Eq. (34). This is one reason why we can obtain the realistic baryon asymmetry in the extended model. Numerically, the resultant asymmetry becomes smaller than Eq. (42) due to the wash-out effect of inverse decay process, as in the standard baryogenesis scenario by the decay of heavy particle.

\section{Thermal history}

In this section, we discuss the thermal history of the universe. We introduce a new scalar $S$ to make the Higgs field stay at 
false vacuum in the early universe, where $S$ is singlet under the SM gauge group. ${ }^{10}$

First, we explain the zero temperature scalar potential of the extended model with $S$ and the thermal correction to it. Then we discuss how the Higgs field is in false vacuum in the early universe.

\subsection{Zero temperature Higgs potential}

The tree level scalar potential is given by

$V_{\text {tree }}(h, S)=-\kappa \frac{m_{S}^{2}}{4 \lambda_{S}} h^{2}+\frac{1}{4} \lambda h^{4}+\kappa h^{2} S^{2}-\frac{1}{2} m_{S}^{2} S^{2}+\lambda_{S} S^{4}$,

where $S$ is the new singlet-scalar field. We consider the region where all couplings take $\mathcal{O}(0.1-1)$ value. Although $\lambda$ becomes small or negative at high scale in the SM (see e.g. Ref. [20]), now the running of $\lambda$ is modified, $\lambda$ can take values $\mathcal{O}(0.1-1)$ since some scalar fields are added.

We note that the one-loop Coleman-Weinberg potential can be safely neglected because of $\mathcal{O}(0.1-1)$ couplings, and therefore we do not include it for simplicity.

The potential (43) has an absolute minimum at ${ }^{11}$

$\langle h\rangle=0, \quad\langle S\rangle=\frac{1}{2} \sqrt{\frac{m_{S}^{2}}{\lambda_{S}}} \equiv v_{S}$.

The quadratic term of the SM Higgs is added in order to make the Higgs massless in this vacuum.

\subsection{Thermal potential}

We follow Ref. [21] and show the thermal potentials. The thermal potentials are evaluated at the one-loop level where the loop effects of the massive Higgs boson, $W, Z$ boson, the top quark and the scalar $S$ are included. For the gauge fields, we employ the Landau gauge where the ghost fields are massless and do not have the $h$ field dependence. The NG bosons $\chi_{i}$ in the Higgs doublet field (4) are neglected since their effects are small.

As the thermal effects, there are two components, namely $V_{\mathrm{FT}}(h, T)$ and $V_{\text {ring }}(h, T) .{ }^{12}$ The main contribution of thermal effects comes from $V_{\mathrm{FT}}(h, T)$, which is

\footnotetext{
$\overline{10}$ In the mechanism we have proposed, it is important that the Higgs field obtains a large expectation value at higher temperature. To realize this situation, we introduce this singlet-scalar field $S$. If one can realize this situation by other ways, we do not have to introduce it. But, naively, introducing the singlet-scalar field is a simplest way.

11 The potential (43) has a minimum at $\langle h\rangle=\sqrt{\frac{\kappa m_{S}^{2}}{2 \lambda \lambda_{S}}}=\sqrt{\frac{2 \kappa}{\lambda}} v_{S}$, $\langle S\rangle=0$. This minimum does not becomes the absolute minimum but the local one for the parameter space we consider here.

12 The derivation of these functions is shown in Appendix A.
}

$$
\begin{aligned}
& V_{\mathrm{FT}}(h, T)=\frac{T^{4}}{2 \pi^{2}}\left[J_{B}\left(\tilde{m}_{S}^{2} / T^{2}\right)+J_{B}\left(\tilde{m}_{h}^{2} / T^{2}\right)\right. \\
& \left.\quad+6 J_{B}\left(m_{W}^{2} / T^{2}\right)+3 J_{B}\left(m_{Z}^{2} / T^{2}\right)-12 J_{F}\left(m_{t}^{2} / T^{2}\right)\right],
\end{aligned}
$$

where the mass for each particle is given by

$$
\begin{aligned}
m_{W}^{2} & =\frac{g_{2}^{2}}{4} h^{2}, \quad m_{Z}^{2}=\frac{g_{2}^{2}+g_{Y}^{2}}{4} h^{2}, \quad m_{t}^{2}=\frac{y_{t}^{2}}{2} h^{2}, \\
\tilde{m}_{h}^{2} & =3 \lambda h^{2}-\kappa \frac{m_{S}^{2}}{2 \lambda_{S}}+2 \kappa S^{2}, \quad \tilde{m}_{S}^{2}=12 \lambda_{S} S^{2}+2 \kappa h^{2}-m_{S}^{2} ;
\end{aligned}
$$

the thermal functions are defined as

$$
\begin{aligned}
& J_{B}\left(r^{2}\right)=\int_{0}^{\infty} \mathrm{d} x x^{2} \ln \left(1-\mathrm{e}^{-\sqrt{x^{2}+r^{2}}}\right), \\
& J_{F}\left(r^{2}\right)=\int_{0}^{\infty} \mathrm{d} x x^{2} \ln \left(1+\mathrm{e}^{-\sqrt{x^{2}+r^{2}}}\right) .
\end{aligned}
$$

Remember here that the coupling constants $g_{2}, g_{Y}$ and $y_{t}$ are $\mathrm{SU}(2)_{L}, \mathrm{U}(1)_{Y}$ and top-Yukawa coupling constants, respectively. Since one cannot analytically and exactly evaluate these functions, the approximated expressions are made. ${ }^{13}$

There are contributions to the ring diagrams (or the daisy diagrams) from the Higgs boson and the gauge boson:

$$
\begin{aligned}
V_{\text {ring }}(h, T)= & -\frac{T}{12 \pi}\left[\left(\tilde{m}_{h}^{2}+\Pi_{h}(T)\right)^{3 / 2}-\tilde{m}_{h}^{3}\right] \\
& -\frac{T}{12 \pi}\left[\left(\tilde{m}_{S}^{2}+\Pi_{h}(T)\right)^{3 / 2}-\tilde{m}_{S}^{3}\right] \\
& -\frac{T}{12 \pi}\left[2 a_{g}^{3 / 2}+\frac{1}{2 \sqrt{2}}\left(a_{g}+c_{g}\right.\right. \\
& \left.-\left[\left(a_{g}-c_{g}\right)^{2}+4 b_{g}^{2}\right]^{1 / 2}\right)^{3 / 2} \\
& +\frac{1}{2 \sqrt{2}}\left(a_{g}+c_{g}+\left[\left(a_{g}-c_{g}\right)^{2}+4 b_{g}^{2}\right]^{1 / 2}\right)^{3 / 2} \\
& \left.-\frac{1}{4}\left[g_{2}^{2} h^{2}\right]^{3 / 2}-\frac{1}{8}\left[\left(g_{2}^{2}+g_{Y}^{2}\right) h^{2}\right]^{3 / 2}\right],
\end{aligned}
$$

where the first and second terms correspond to the contribution from the Higgs and the scalar $S ;{ }^{14}$ the thermal masses of the Higgs and scalar $S$ are

13 The high temperature expansion is often used. However, they are not useful for the case where we see the large field value of $h$. Therefore, the fitting functions (A23) are also employed [22]. See Appendix A for details.

${ }^{14}$ Combining the ring contribution of the Higgs boson and the first term of Eq. (45), we can write

$$
\begin{aligned}
& \frac{T^{4}}{2 \pi^{2}} J_{B}\left(\tilde{m}_{h}^{2} / T^{2}\right)-\frac{T}{12 \pi}\left[\left(\tilde{m}_{h}^{2}+\Pi_{h}(T)\right)^{3 / 2}-\tilde{m}_{h}^{3}\right] \\
& \quad=\frac{T^{4}}{2 \pi^{2}} J_{B}\left(\tilde{m}_{h}^{2}(T) / T^{2}\right),
\end{aligned}
$$


$\Pi_{h}(T)=\frac{T^{2}}{12}\left(\frac{9}{4} g_{2}^{2}+\frac{3}{4} g_{Y}^{2}+3 y_{t}^{2}+6 \lambda+2 \kappa\right)$,

$\Pi_{S}(T)=T^{2}\left(\frac{\lambda_{S}}{4}+\frac{2 \kappa}{3}\right) ;$

and we have defined

$a_{g}=\frac{1}{4} g_{2}^{2} h^{2}+\frac{11}{6} g_{2}^{2} T^{2}, \quad b_{g}=-\frac{1}{4} g_{2} g_{Y} h^{2}$,

$c_{g}=\frac{1}{4} g_{Y}^{2} h^{2}+\frac{11}{6} g_{Y}^{2} T^{2}$.

To summarize, in order to trace the thermal history of the Higgs potential in the SM, we analyze the effective potential,

$V_{\text {eff }}(h, S, T)=V_{\text {tree }}(h, S)+V_{\mathrm{FT}}(h, S, T)+V_{\text {ring }}(h, S, T)$,

where $V_{\text {tree }}(h, S)$ is given in Eq. (43). In next subsection, we investigate the phase transition of Higgs field by using this potential.

\subsection{Thermal history}

In the early universe, due to the finite temperature effect, $S$ and $H$ do not have the vacuum expectation value (VEV). ${ }^{15}$ They develop their respective VEVs at the temperature when the thermal mass term becomes comparable with their negative mass term. By utilizing the high temperature expansion (A21) and (A22), we estimate the critical temperatures which are given as the vanishing curvature of $V_{\text {eff }}(h, S, T)$ at the origin $(h, S)=(0,0)$, namely

$$
\begin{aligned}
& \left.\frac{\partial^{2} V_{\text {eff }}\left(h, S, T_{S}\right)}{\partial S^{2}}\right|_{h=0, S=0}=0, \\
& \left.\frac{\partial^{2} V_{\text {eff }}\left(h, S, T_{h}\right)}{\partial h^{2}}\right|_{h=0, S=0}=0 .
\end{aligned}
$$

Solving these equations for $T$, we find ${ }^{16}$

$$
\begin{aligned}
& T_{S}=\frac{2 \sqrt{3} \sqrt{2 v_{s}^{2} \lambda_{S}}}{\sqrt{\kappa+6 \lambda_{S}}}, \\
& T_{h}=\frac{4 \sqrt{6} \sqrt{v_{s}^{2} \kappa}}{\sqrt{9 g_{2}^{2}+3 g_{Y}^{2}+12 y_{t}^{2}+8 \kappa+12 \lambda}} .
\end{aligned}
$$

Footnote 14 continued

where $\tilde{m}_{h}^{2}(T)=\tilde{m}_{h}^{2}+\Pi_{h}(T)$ is the Debye mass of the Higgs boson. In the same manner, the thermal effects for the scalar $S$ also can be written as the same form.

15 Our thermal scenario is similar to Ref. [23], where the gravitational wave from electroweak phase transition at the high scale is discussed.

16 Here the $V_{\text {ring }}$ contribution is neglected. This should still provide an approximate estimation of the phase transition temperature.
Here $T_{S}$ and $T_{h}$ denote the critical temperatures of the phase transition of $S$ and $h$, respectively. Our scenario is as follows. The phase transition of Higgs field happens at $T=T_{h}$. At this time, $S$ and $h$ are in the false vacuum, $\langle S\rangle=0,\langle h\rangle=\sqrt{\frac{2 \kappa}{\lambda}} v_{S}$, and the lepton number is created by the decay of heavy neutrinos. After that, at $T=T_{S}, S$ develops $\operatorname{VEV}\langle S\rangle=v_{S}$, and then $\langle h\rangle$ comes back to the true vacuum Eq. (44).

In order to work with our scenario, we require

$T_{S}<T_{h}$

Moreover, $S$ must have a negative mass at $\langle S\rangle=0,\langle h\rangle=$ $\sqrt{\frac{2 \kappa}{\lambda}} v_{S}$, namely $\tilde{m}_{S}<0$, which yields

$\lambda_{S}>\frac{\kappa^{2}}{\lambda}$

As an example of successful parameters, we take $\kappa=$ $0.7, \lambda_{S} \simeq 1.5, \lambda=0.4$ and $\langle h\rangle=2 \times 10^{13} \mathrm{GeV} . T_{h}$ and $T_{S}$ become

$T_{S} \simeq 1.9 v_{S}, \quad T_{h} \simeq 2.0 v_{S}$,

and Eq. (57) is satisfied. Here $g_{Y}=g_{2}=y_{t}=0.5$ is used.

Therefore, by solving the Boltzmann equations with

$z_{\text {ini }}=\frac{M_{v}}{T_{h}}, \quad z_{\text {final }}=\frac{M_{v}}{T_{S}}$

we can calculate the asymmetry. For example, we obtain ${ }^{17}$

$N_{B-L} \simeq 7.0 \times 10^{-7}$

with the parameter set Eq. (41). Here we have taken into account the wash-out factor [15] in the symmetric phase,

$\exp \left[-T_{S} / 2 \times 10^{13} \mathrm{GeV}\right]$

This implies that we can realize the observed value, $N_{B-L \text {,obs }}$ $=4.8 \times 10^{-8}$, by slightly changing the value of $C P$ phase. We notice that a numerical study is necessary to establish which values of the couplings return an acceptable pattern of symmetry breaking, as currently approximate estimates are provided in the paper.

Finally, let us briefly discuss the validity of the effective Lagrangian Eq. (3). The temperature of the phase transition is

$T_{h} \simeq \sqrt{\frac{2 \lambda}{\kappa}}\langle h\rangle \simeq 2 \times 10^{13} \mathrm{GeV}$,

${ }^{17}$ Here we take the thermal initial condition, $N_{X}=3 / 4, N_{B-L}=0$. 
while the cutoff scale in Eq. (3) is Eq. (6). It can be seen that, as long as $m_{v} \lesssim 0.1 \mathrm{eV}, T_{h}$ is much smaller than $\Lambda$ in Eq. (41). Although $T_{h}$ is close to $\Lambda_{2}$ and $\Lambda_{3}$, it is still below these cutoffs. Hence, the effective Lagrangian would be valid in this region.

\section{Summary and discussion}

We have considered the possibility of baryogenesis in a false vacuum where the Higgs field develops a large field value compared with the electroweak scale. Since all the SM particles receive mass from the coupling with the Higgs boson, the large field value of the Higgs field means that they are super-heavy. We have estimated the asymmetry produced by the decay of the heavy left-handed neutrino. It has turned out that the decay of the neutrino cannot realize the observed baryon asymmetry. If the new higher-dimensional operators are introduced, the decay of the neutrino can provide sufficient asymmetry.

We have also presented the thermal history where the Higgs field develops a large value in the early universe. It has been found that, by adding the singlet scalar $S$, our scenario safely works.

Finally, we briefly mention the possibility of the high scale electroweak baryogenesis. So far, we pursued the possibility that the baryon asymmetry is created by the heavy particle, while the lepton number violation is given by Majorana mass term of the left-handed neutrino. However, if the coupling $\lambda$ is small, the electroweak phase transition at high scale becomes of first order. Since our extended model has many $C P$ phases, there is a possibility to generate the $B+L$ asymmetry. If the $L$ asymmetry is washed out in the false vacuum, the net $B$ asymmetry survives. The condition of the $L$ wash-out would be roughly given by

$\left(\frac{\langle h\rangle}{\Lambda}\right)^{2} \frac{1}{8 \pi} M_{W} \gtrsim \sqrt{10} \frac{T^{2}}{M_{P}}$

By putting $T \simeq\langle h\rangle, M_{W} \simeq\langle h\rangle$, we obtain

$\langle h\rangle \gtrsim 1 \times 10^{13} \mathrm{GeV}$.

Hence, we have a possibility to create the baryon asymmetry by the electroweak baryogenesis in addition to the decay of heavy particle.

Acknowledgements YH is supported by Japan Society for the Promotion of Science (JSPS) Fellowships for Young Scientists. We thank Hikaru Kawai for important discussion of the phase diagram of the standard model. We also thank Ryusuke Jinno and Masahiro Takimoto for useful comments on the Higgs thermal potential.

Open Access This article is distributed under the terms of the Creative Commons Attribution 4.0 International License (http://creativecomm ons.org/licenses/by/4.0/), which permits unrestricted use, distribution, and reproduction in any medium, provided you give appropriate credit to the original author(s) and the source, provide a link to the Creative Commons license, and indicate if changes were made.

Funded by $\mathrm{SCOAP}^{3}$.

\section{Appendix: The effective potential at finite temperature}

In this appendix, following Ref. [21], we show the derivation of the thermal effects on the Higgs potential in the SM. We consider the one-loop contribution of a particle with the mass $m(h)$ to the potential, which typically has the following form:

$V_{\text {lloop }}(h, T)= \pm \frac{1}{2} \operatorname{Tr} \ln \left(k^{2}+m^{2}(h)\right)$,

where " $\operatorname{Tr}$ " denotes the functional trace; $k$ is the Euclidean momentum; the boson (fermion) loop case has overall positive (negative) sign. For a particle with one degree of freedom, the potential is

$V_{1 \text { loop }}(h, T)= \pm \frac{1}{2} \int \frac{\mathrm{d}^{4} k}{(2 \pi)^{4}} \ln \left(k^{2}+m^{2}(h)\right)$.

At finite temperature, the time direction of momentum is discretized and its loop integral changes to the Matsubara summation:

$\int \frac{\mathrm{d} k_{0}}{2 \pi} \int \frac{\mathrm{d}^{3} k}{(2 \pi)^{3}} f\left(k_{0}, \vec{k}\right)=T \sum_{n=-\infty}^{\infty} \int \frac{\mathrm{d}^{3} k}{(2 \pi)^{3}} f\left(\omega_{n}, \vec{k}\right)$,

with the Matsubara frequency,

$\omega_{n}= \begin{cases}2 n \pi T & \text { for bosons, } \\ (2 n+1) \pi T & \text { for fermions. }\end{cases}$

Therefore, Eq. (A2) can be calculated as

$$
\begin{aligned}
V_{\text {1loop }}(h, T) & = \pm \frac{T}{2} \int \frac{\mathrm{d}^{3} k}{(2 \pi)^{3}}\left[\beta \omega+2 \ln \left(1 \mp e^{-\beta \omega}\right)\right] \\
& = \pm \frac{1}{2} \int \frac{\mathrm{d}^{3} k}{(2 \pi)^{3}} \omega \pm T \int \frac{\mathrm{d}^{3} k}{(2 \pi)^{3}} \ln \left(1 \mp \mathrm{e}^{-\beta \omega}\right),
\end{aligned}
$$

where $\omega=\sqrt{\vec{k}^{2}+m^{2}}$; the sign (+) and (-) in the logarithm apply to fermions and bosons, respectively. The first term does not depend on temperature and is rewritten as

$$
\begin{aligned}
V_{\mathrm{CW}}(h) & \equiv \pm \frac{1}{2} \int \frac{\mathrm{d}^{3} k}{(2 \pi)^{3}} \omega \\
& = \pm \frac{1}{2} \int \frac{\mathrm{d}^{4} k}{(2 \pi)^{4}} \ln \left(k^{2}+m^{2}\right)
\end{aligned}
$$




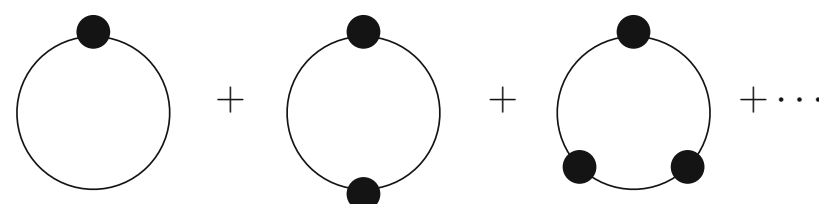

Fig. 3 The ring diagrams. The black circle denotes the propagator with loop corrections

This is one-loop contribution at vanishing temperature, i.e. the Coleman-Weinberg potential. The second term is the thermal potential at one-loop level and becomes

$V_{\mathrm{FT}}(h, T) \equiv \pm \frac{T}{2 \pi^{2}} \int \mathrm{d} k k^{2} \ln \left(1 \pm \mathrm{e}^{-\beta \omega}\right)= \pm \frac{T^{4}}{2 \pi^{2}} J_{B(F)}\left(r^{2}\right)$,

where the thermal functions for boson and fermion are defined as

$J_{B}\left(r^{2}\right)=\int_{0}^{\infty} \mathrm{d} x x^{2} \ln \left(1-\mathrm{e}^{-\sqrt{x^{2}+r^{2}}}\right)$,

$J_{F}\left(r^{2}\right)=\int_{0}^{\infty} \mathrm{d} x x^{2} \ln \left(1+\mathrm{e}^{-\sqrt{x^{2}+r^{2}}}\right)$,

with $x \equiv|\vec{k}| / T$ and $r \equiv m(h) / T$. Note that in general case, the operator $k^{2}+m^{2}(h)$ is not diagonal, i.e. $k^{2} \delta_{i j}+m_{i j}^{2}(h)$. Therefore, the mass matrix $m_{i j}^{2}(h)$ has to be diagonalized.

In the SM case, taking account of the degrees of freedom of particles, the thermal potential is given by

$$
\begin{aligned}
V_{\mathrm{FT}}(h, T)= & \sum_{i=W, Z, h} n_{i} \frac{T^{4}}{2 \pi^{2}} J_{B}\left(\left(m_{i}^{B}(h) / T\right)^{2}\right) \\
& -\sum_{i=t} n_{i} \frac{T^{4}}{2 \pi^{2}} J_{F}\left(\left(m_{i}^{F}(h) / T\right)^{2}\right),
\end{aligned}
$$

where $n_{W}=6, n_{Z}=3, n_{t}=12$ and $n_{h}=1$.

Next, we consider the ring (or daisy) contributions shown in Fig. 3, which are the next-higher-order corrections and are related to the infrared divergence; see e.g. [24] for a detailed discussion. The ring contribution for the Higgs field is given by

$$
\begin{aligned}
V_{\text {ring }}^{\text {Higgs }}(h, T) \equiv & -\frac{1}{2} T \sum_{n=-\infty}^{\infty} \int \frac{\mathrm{d}^{3} k}{(2 \pi)^{3}} \\
& \times \sum_{l=1}^{\infty} \frac{1}{l}\left(-\frac{1}{\omega_{n}^{2}+\vec{k}^{2}+m_{h}^{2}(h)} \Pi_{h}(T)\right)^{l} \\
= & -\frac{T}{12 \pi} \operatorname{Tr}\left\{\left[m_{h}^{2}(h)+\Pi_{h}(T)\right]^{3 / 2}-m_{h}^{3}(h)\right\},
\end{aligned}
$$

where the thermal mass comes from the diagrams in the limit $m(h) / T \ll 1$ shown in Fig. 4 and becomes

$$
\begin{aligned}
\Pi_{h}(T) & =\Pi_{\phi}^{\left(A_{\mu}^{a}\right)}(T)+\Pi_{\phi}^{\left(B_{\mu}\right)}(T)+\Pi_{\phi}^{(\psi)}(T)+\Pi_{\phi}^{(\phi)}(T) \\
& =\frac{T^{2}}{12}\left(\frac{9}{4} g_{2}^{2}+\frac{3}{4} g_{Y}^{2}+3 y_{t}^{2}+6 \lambda\right),
\end{aligned}
$$

with

$$
\begin{aligned}
\Pi_{\phi}^{\left(A_{\mu}^{a}\right)}(T) & =\frac{3}{16} g_{2}^{2} T^{2}, \quad \Pi_{\phi}^{\left(B_{\mu}\right)}(T)=\frac{1}{16} g_{Y}^{2} T^{2}, \\
\Pi_{\phi}^{(\psi)}(T) & =\frac{1}{4} y_{t}^{2} T^{2}, \quad \Pi_{\phi}^{(\phi)}(T)=\frac{1}{2} \lambda T^{2} .
\end{aligned}
$$

Note that these contributions are evaluated by setting the external momentum to zero since we are interested in the infrared limit.

In a similar manner, one can obtain the ring contributions from the gauge bosons, which becomes

$V_{\text {ring }}^{\mathrm{gb}}(h, T) \equiv-\frac{T}{12 \pi} \operatorname{Tr}\left\{\left[M^{2}(h)+\Pi_{00}(T)\right]^{3 / 2}-M^{3}(h)\right\}$.

Here the mass matrices in the original gauge field basis $\left(A_{\mu}^{i}, B_{\mu}\right)$ are

$$
M^{2}(h)=\left(\begin{array}{cccc}
g_{2}^{2} h^{2} / 4 & 0 & 0 & 0 \\
0 & g_{2}^{2} h^{2} / 4 & 0 & 0 \\
0 & 0 & g_{2}^{2} h^{2} / 4 & -g_{Y} g_{2} h^{2} / 4 \\
0 & 0 & -g_{Y} g_{2} h^{2} / 4 & g_{2}^{2} h^{2} / 4
\end{array}\right)
$$

$\Pi_{00}(T)=\left(\begin{array}{cccc}\Pi_{00}^{(2)}(T) & 0 & 0 & 0 \\ 0 & \Pi_{00}^{(2)}(T) & 0 & 0 \\ 0 & 0 & \Pi_{00}^{(2)}(T) & 0 \\ 0 & 0 & 0 & \Pi_{00}^{(1)}(T)\end{array}\right)$,

where $\Pi_{00}(T)$ is the (00) component of the polarization tensor in the infrared limit, namely $\Pi_{\mu \nu}(p=0, T)$ and

$\Pi_{00}^{(1)}(T)=\Pi_{\phi}^{(1)}(T)+\Pi_{\psi}^{(1)}(T)=\frac{11}{6} g_{Y}^{2} T^{2}$,

$\Pi_{00}^{(2)}(T)=\Pi_{\mathrm{gb}}^{(2)}(T)+\Pi_{\phi}^{(2)}(T)+\Pi_{\psi}^{(2)}(T)=\frac{11}{6} g_{2}^{2} T^{2}$,

with

$$
\begin{array}{ll}
\Pi_{\phi}^{(1)}(T)=\frac{1}{6} g_{Y}^{2} T^{2}, & \Pi_{\psi}^{(1)}(T)=\frac{5}{3} g_{Y}^{2} T^{2}, \\
\Pi_{\mathrm{gb}}^{(2)}(T)=\frac{2}{3} g_{2}^{2} T^{2}, & \Pi_{\phi}^{(2)}(T)=\frac{1}{6} g_{2}^{2} T^{2},
\end{array}
$$

These thermal masses are obtained by calculating the twopoint functions of $\mathrm{SU}(2)$ and $\mathrm{U}(1)$ gauge fields shown in Fig. 5. Evaluating the eigenvalues of $M^{2}(h)+\Pi_{00}(T)$ and 


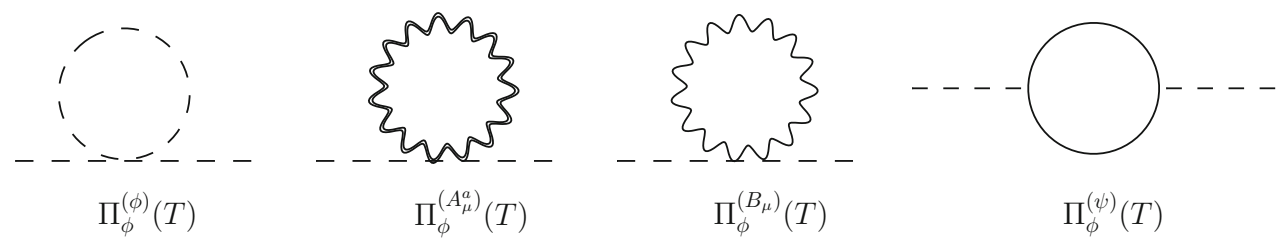

Fig. 4 The two-point functions of Higgs field at one-loop level which yield the thermal masses. The dashed, single wave, double wave and solid lines denote the Higgs, $\mathrm{U}(1)_{Y}$ gauge, $\mathrm{SU}(2)_{L}$ and top quark, respectively,

Fig. 5 The two-point functions of $\mathrm{SU}(2)$ and $\mathrm{U}(1)$ gauge fields at one-loop level which yield the thermal masses. The dashed, single wave, double wave, solid and dot lines denote the Higgs, $\mathrm{U}(1)_{Y}$ gauge, $\mathrm{SU}(2)_{L}$, top quark and ghost, respectively
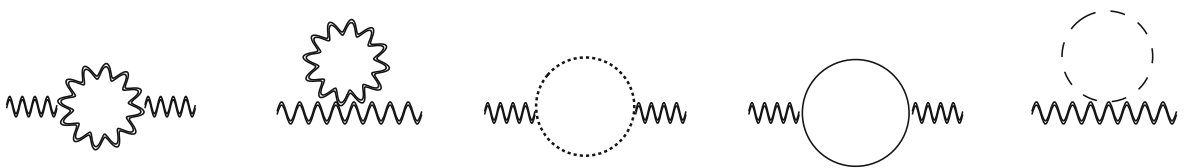

$\Pi_{\mathrm{gb}}^{(2)}(T)$

$\Pi_{\psi}^{(2)}(T)$

$\Pi_{\phi}^{(2)}(T)$
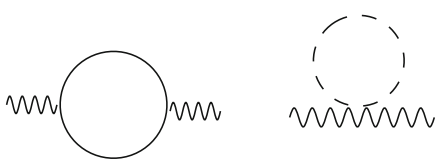

$\Pi_{\psi}^{(1)}(T)$

$\Pi_{\phi}^{(1)}(T)$
$M^{2}(h)$ to the three-half power, and then taking trace of them, we have

$$
\begin{aligned}
V_{\text {ring }}^{\mathrm{gb}}(h, T)= & -\frac{T}{12 \pi}\left[2 a_{g}^{3 / 2}+\frac{1}{2 \sqrt{2}}\left(a_{g}+c_{g}\right.\right. \\
& \left.-\left[\left(a_{g}-c_{g}\right)^{2}+4 b_{g}^{2}\right]^{1 / 2}\right)^{3 / 2} \\
& +\frac{1}{2 \sqrt{2}}\left(a_{g}+c_{g}+\left[\left(a_{g}-c_{g}\right)^{2}+4 b_{g}^{2}\right]^{1 / 2}\right)^{3 / 2} \\
& \left.-\frac{1}{4}\left[g_{2}^{2} h^{2}\right]^{3 / 2}-\frac{1}{8}\left[\left(g_{2}^{2}+g_{Y}^{2}\right) h^{2}\right]^{3 / 2}\right], \quad \text { (A20) }
\end{aligned}
$$

where $a_{g}, b_{g}$ and $c_{g}$ are given in Eq. (52).

Note that we have worked in the Landau gauge to evaluate the contributions from the gauge bosons. Although the thermal mass matrix (A14) can be diagonal only in the limit $m_{W}(h) / T \ll 1$ and $m_{Z}(h) / T \ll 1$, the ring contribution is still valid for the larger mass, thus the larger field value than temperature. This is because the ring contribution vanishes for the larger mass.

In case where the scalar $S$ is introduced, the contribution from the diagram shown in Fig. 6 is added, and then the thermal masses of the Higgs field and $S$ are given as Eqs. (50) and (51), respectively.

\subsection{The thermal functions and their approximation}

The high temperature expansion is often applied to the thermal functions (A8). However, it is not useful for investigat-

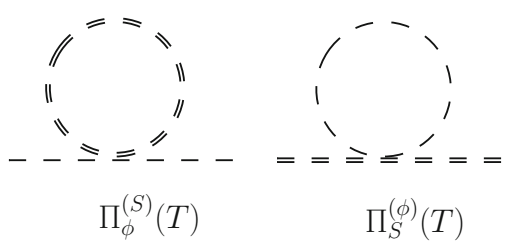

Fig. 6 The diagrams which contribute to the thermal masses of the Higgs field and the new scalar one. The double dashed line denotes the new scalar field $S$

ing the large field value $m(\phi) / T \equiv r \geq 1$. In this subsection we compare the exact forms of the thermal functions (A8) numerically evaluated with their approximated forms and investigate the effectiveness of them.

The thermal functions (A8) with the high temperature expansion become

$$
\begin{aligned}
J_{B}\left(r^{2}\right) \simeq & -\frac{\pi^{4}}{45}+\frac{\pi^{2}}{12} r^{2}-\frac{\pi}{6} r^{3} \\
& -\frac{r^{4}}{32}\left[\ln \left(r^{2} / 16 \pi^{2}\right)+2 \gamma_{E}-\frac{3}{2}\right], \\
J_{F}\left(r^{2}\right) \simeq & \frac{7 \pi^{4}}{360}-\frac{\pi^{2}}{24} r^{2}-\frac{r^{4}}{32}\left[\ln \left(r^{2} / \pi^{2}\right)+2 \gamma_{E}-\frac{3}{2}\right],
\end{aligned}
$$

where $\gamma_{E} \approx 0.57721$ is the Euler gamma. Besides, it is known that the thermal functions can be fitted by the following functions [22]: 


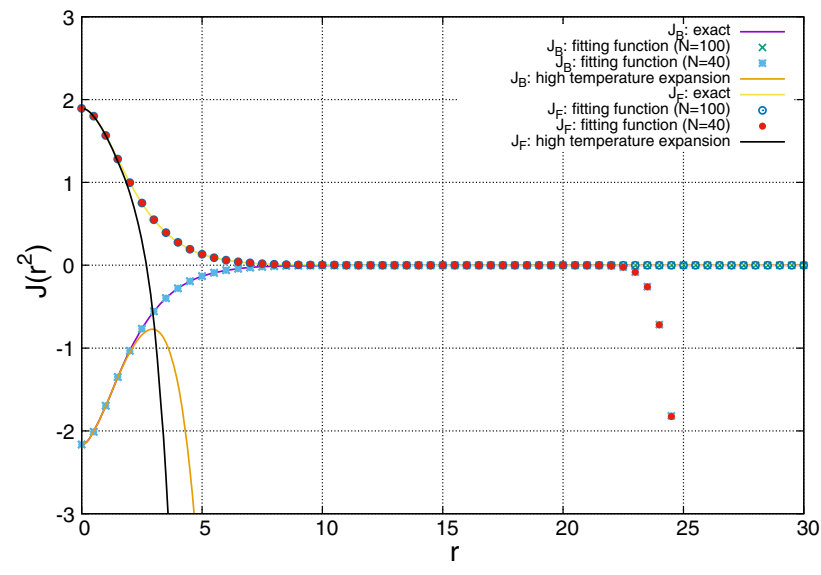

Fig. 7 Comparison of the thermal functions between different approximations

$J_{B(F)}\left(r^{2}\right)=\mathrm{e}^{-r} \sum_{n=0}^{N_{B(F)}} c_{n}^{B(F)} r^{n}$,

where $N_{B(F)}$ and $c_{n}^{B(F)}$ are the truncation order of the series and the fitting coefficients, respectively. For the fixed truncation order $N_{F(B)}$, we find the coefficients $c_{n}^{F(B)}$ by fitting the exact results numerically evaluated.

We show the comparisons between results of exact form Eq. (A8) and the approximated forms Eqs. (A21)-(A23) in Fig. 7. We see that the high temperature expansions are actually valid for $r \leq 2$ and the fitting functions with $N_{B(F)}=40$ break down for $r>22$. The fitting functions with $N_{B(F)}=100$ are valid for large value of $r$. Therefore, it is useful for evaluating a potential of large field values since $r=m(\phi) / T$ and the mass $m(\phi)$ is proportional to the value of the field $\phi$.

\section{References}

1. ATLAS, G. Aad et al., Observation of a new particle in the search for the Standard Model Higgs boson with the ATLAS detector at the LHC. Phys. Lett. B 716, 1-29 (2012). arXiv:1207.7214

2. CMS, S. Chatrchyan et al., Observation of a new boson at a mass of $125 \mathrm{GeV}$ with the CMS experiment at the LHC. Phys. Lett. B 716, 30-61 (2012). arXiv:1207.7235
3. P.A.R. Ade et al., Planck 2015 results. XIII. Cosmological parameters. Astron. Astrophys. 594, A13 (2015), arXiv:1502.01589

4. A.D. Sakharov, Violation of CP invariance, c asymmetry, and Baryon asymmetry of the universe. Pisma Zh. Eksp. Teor. Fiz. 5, 32-35 (1967) [Usp. Fiz. Nauk 161, 61 (1991)]

5. M. Yoshimura, Unified gauge theories and the Baryon number of the universe. Phys. Rev. Lett. 41, 281-284 (1978) [Erratum: Phys. Rev. Lett. 42, 746 (1979)]

6. I. Affleck, M. Dine, A new mechanism for baryogenesis. Nucl. Phys. B 249, 361-380 (1985)

7. M. Fukugita, T. Yanagida, Baryogenesis without grand unification. Phys. Lett. B 174, 45-47 (1986)

8. W. Buchmuller, R.D. Peccei, T. Yanagida, Leptogenesis as the origin of matter. Ann. Rev. Nucl. Part. Sci. 55, 311-355 (2005). arXiv:hep-ph/0502169

9. C.S. Fong, E. Nardi, A. Riotto, Leptogenesis in the universe. Adv. High Energy Phys. 2012, 158303 (2012). arXiv:1301.3062

10. R.F. Dashen, B. Hasslacher, A. Neveu, Nonperturbative methods and extended hadron models in field theory. 3. Four-dimensional nonabelian models. Phys. Rev. D 10, 4138 (1974)

11. N.S. Manton, Topology in the Weinberg-Salam theory. Phys. Rev. D 28, 2019 (1983)

12. F.R. Klinkhamer, N.S. Manton, A saddle point solution in the Weinberg-Salam theory. Phys. Rev. D 30, 2212 (1984)

13. V.A. Kuzmin, V.A. Rubakov, M.E. Shaposhnikov, On the anomalous electroweak baryon number nonconservation in the early universe. Phys. Lett. B 155, 36 (1985)

14. H. Aoki, H. Kawai, String scale baryogenesis. Prog. Theor. Phys. 98, 449-456 (1997). arXiv:hep-ph/9703421

15. Y. Hamada, K. Kawana, Reheating-era leptogenesis. Phys. Lett. B 763, 388-392 (2016). arXiv: 1510.05186

16. E.W. Kolb, S. Wolfram, Baryon number generation in the early universe. Nucl. Phys. B 172, 224 (1980) [Erratum: Nucl. Phys. B 195, $542(1982)]$

17. E.W. Kolb, M.S. Turner, The early universe. Front. Phys. 69, 1-547 (1990)

18. Particle Data Group, C. Patrignani et al., Review of particle physics. Chin. Phys. C 40(10), 100001 (2016)

19. Y. Hamada, H. Kawai, K.-Y. Oda, Minimal Higgs inflation. PTEP 2014, 023B02 (2014). arXiv: 1308.6651

20. Y. Hamada, H. Kawai, K.-Y. Oda, Bare Higgs mass at Planck scale. Phys. Rev. D 87(5), 053009 (2013). arXiv: 1210.2538

21. M.E. Carrington, The effective potential at finite temperature in the Standard Model. Phys. Rev. D 45, 2933-2944 (1992)

22. K. Funakubo, E. Senaha, Electroweak phase transition, critical bubbles and sphaleron decoupling condition in the MSSM. Phys. Rev. D 79, 115024 (2009). arXiv:0905.2022

23. R. Jinno, K. Nakayama, M. Takimoto, Gravitational waves from the first order phase transition of the Higgs field at high energy scales. Phys. Rev. D 93(4), 045024 (2016). arXiv:1510.02697

24. J.I. Kapusta, C. Gale, Finite-Temperature Field Theory: Principles and Applications (Cambridge University Press, Cambridge, 2011) 